PURULENT PERICARDITIS WITH REPORT OE A CASE DLE TO TRAUMATISM.

Read before the Michigan state Medical tociety, Graud Rapids, Mich. May 14, 18:97

BY FRANK W. GARBER, B.S., M.D. MCSKEGON, MICH.

Purulent pericarditis presents essentially the same chain of symptoms as those shown by a non-septic pericardial inflammation. The history of the case may point strongly in the direction of a purulent effusion, but the aspirator alone can determine positively the nature of the fluid. The disease may even run its course without such aids to diagnosis, as fever and edema of overlying tissue. A preëxistent or a co-existing ulcerative endocarditis, a pyemia, tuberculosis or an empyema, an abscess of the mediastinum, caries of the ribs, gangrene of the lungs, or a trauma may make evident the presence of pus, and are among the leading causes of purulent effusion into the pericardium. Pericarditis whether purulent or non-purulent is a secondary trouble, and is probably frequently overlooked by the physician.

The healthy pericardial sac of an adult holds from 420 to 630 grams. It is somewhat pyriform in shape and lies back of and to the left of the sternum, extending from the third to the seventh ribs with its broadest portion at its diaphragmatic attachment. Upon the greater or less distension of this sac, together with the intensity of the inflammation and the character of the exudate, depend the phenomena of pericarditis. As effusion takes place, the sac gradually distends from below upward, the area of precordial dulness broadening and rising as the quantity of fluid increases. The lungs are crowded back and to either side. The diaphragm is depressed. The dulness may extend from the xiphoid process to the top of the sternum, and from nipple to nipple. As the amount of effusion increases, the site of the heart's impulse is raised and carried outward, sometimes reaching as high as the third interspace. With encroachment upon the lung space come dyspnea, and an interference with the normal respiratory movements. The play of the upper ribs is increased and there is limited motion on the left. The lateral movements of the abdomen are lessened, the play of the left side being less than that of the right. Pressure upon the vena cava and the pulmonary veins tends to venous engorgement, and the dusky countenance so frequently seen. In the young, and where there are large accumulations of fluid there may be precordial prominence. There may be friction sound, and an intermission of the radial pulse during inspiration, at a time when there is no intermittent heart action. Accentuation of the pulmonary second sound is remarked as one of the most constant signs of pericarditis. If to the symptoms enumerated there are added hectic, sweating, and such other familiar signs of confined pus as are characterized by empyema, the diagnosis of purulent pericarditis becomes reasonably certain.

As far back as the seventeenth century, we find clinicians discussing the advisability of opening the pericardial sac for the relief of pericardial effision. It was not until 1819, that the operation was first successfully performed. Romero of Barcelona operated by incision, in three cases, two of which recovered. In 1840 paracentesis was performed by Schuh for pericarditis due to cancer, the patient living six months after the operation. The first instance of tapping of the pericardium, in this country, occurred in the practice of Dr. Warren of Boston, in 1852. These operations were all for the removal of non-purulent effusions, though tapping has been recommended and practiced until recent years for the relief of either class of cases.

The first authentic case I have been able to find of the treatment of purulent pericarditis by the scientific and reasonable method of incision and drainage, is found in the fifth number of the Berliner klin. Wochenschrift of 1881.

A boy ten years of age had suffered from empyema which failed of relief by tapping. Indications pointed to a pericarditis, which by aspiration was found to be purulent. Though several ounces of pus were removed by this method, no improvement followed, but rather. increasing dyspnea, lividity and edema of feet and legs. Sleep became broken and the general condition poor. An incision into the pericardial sac, between the fourth and fifth ribs near the border of the sternum, was then made under the antiseptic precautions then in vogue. Two drainage tubes were placed in the opening and the wound inclosed in a Lister dressing. Prompt relief followed though recovery was retarded by co-existing empyema, which was finally relieved by incision and drainage.

Operation by incision and drainage was performed for the first time in England by Dr. Samuel West in 1883. Great success followed the operation. There was no deformity of the chest and only a very small scar remained.

In 1885, Michaeloff, after a preliminary tapping which was followed by slight improvement, incised the pericardium of an adult female in whom the purulent pericarditis seems to have been a sequela of Bright's disease. Temporary improvement followed, but the patient died a short time after the operation. Postmortem revealed a fatty degeneration of the heart with great dilatation.

In the same year, Gussenbauer resected five ribs and washed out the pericardial sac with a thymol solution. His patient recovered.

Davidson reported in the British Medical Journal, March 1891, two cases treated by incision and drain. age. The first case was that of a boy of six in whom pyopericardium was secondary to necrosis of one of the metatarsal bones, and further complicated by pneumonia and a subperiosteal abscess of the eighth rib. A free incision was made in the fifth interspace, and eight ounces of pus were evacuated. There was some temporary relief but the patient died within a week. The second case occurred in a boy of seven, and followed a purulent pleuritic effusion which had been appropriately treated without giving any apparent relief. Examination revealed the presence of a pericarditis which proved to be suppurative. The sac was opened, free drainage established and the patient made an uninterrupted recovery.

Beekman and Stall report a case in which the peri. cardium was opened after trephining the sternum. Recovery followed.

Bronner relates a case of pericardiotomy in a girl of 11. which terminated fatally after twenty-five days. Scott reported a case of pyopericardium (New Zealand Medical Journal, July 1891), in which he opened the sac, removed a large quantity of pus and irrigated the cavity with a solution of carbolic acid (15 to 1000). The operation was followed by complete recovery.

In the International Medical Magazine, June 189:3, 
Edwards gives the details of a case treated by incision and drainage. The patient was a poorly nourished and illy developed child of 6 who for a month had complained of pains in the left costo-sternal region. There were continuous fever, dulness over the precordial region, dyspnea, edema of face and ankles, and albuminuria. The operation was followed two weeks later by a purulent pleuritic effusion, the relief of which gave no signs of improvement, and the child died of exhaustion at the end of the third week.

Eiselberg of Vienna, reports a case which is of especial interest in connection with this paper, because like my own case, it was traumatic. A boy of seventeen received a stab wound which penetrated the pericardium. Pericarditis followed. Relief from the distressing symptoms was sought by tapping, but without permanent improvement. The cartilage of the fourth rib was accordingly resected, the pericardium exposed, and about two liters of sero-purulent fluid evacuated. The sac was irrigated, the borders of the sac stitched to the edges of the wound, and two drainage tubes inserted. The boy had completely recovered at the expiration of a month. Eiselberg insists on the necessity of stitching the pericardium to the lips of the wound to prevent infection of the pleura, a procedure which seems to have some theoretical warrant, but which in fact seems hardly necessary, as the danger of entering the pleural cavity through the fifth interspace at the point of election is very remote. If we draw a line from the left nipple to the end of the xiphoid process, bisect the line, and with this point of bisection as a center inscribe a circle with a radius of one inch, we shall inclose within this area a portion of the pericardium which in health is not covered by the pleural sac. This area is greatly increased in pericardial effusion.

The following are the details of my own case, the happy termination of which makes the indications for treatment in this class of cases none the more positive though encouraging, perhaps, to larger fidelity to fixed surgical principles, no matter what part is involved.

M. H., a healthy well-developed girl, aged 21 years, in a scuffle with some companions, ran a steel crochet needle into her chest about an inch to the left of the sternum and in the fifth interspace. The needle passed into the tissue, one and a fourth inches, and broke off. The accident occurred in the evening of Jan. 12, 1897, and the patient came to me within a few hours following. She was somewhat nervous, but apparently suffered no pain, while there was only moderate acceleration of the pulse. A small point showing the place of entrance of the needle was all that could be seen. Palpation did not reveal anything and an aseptic incision was made to a considerable distance into the tissue without disclosing anything. I therefore concluded that the broken end of the needle had not remained in the tissue, but had been lost in the excitement following the accident. Acting on this belief, the patient was sent home, with instructions to return again. The search of the following day convinced me that the needle was still there and the patient was advised to have an anesthetic and such operation as was necessary to relieve her. Her consent to such procedure could not be obtained however, until three days from the time of the accident had elapsed. There was at that time considerable rise of temperature and an increased pulse rate. A crucial incision was made, having the point of entrance of the needle, the point of intersection of the lines of incision. The flaps were dissected back, and; after considerable search the end of the needle was found in the intercostal muscle flush with the costal cartilage. It required considerable effort to remove it, and it was found that the hook of the needle had carried a thread of cloth into the pericardium, into which it had entered and in which the needle had remained ninety-six hours. Septic pericarditis was feared, as there was already a temperature of $10 \mathrm{l}$ degrees and a pulse of 125 . It was not thought justifiable at this time, to incise the pericardium. For the twenty-four hours following the removal of the needle, the patient's condition remained much the same, temperature and pulse not declining. On January 17 , two days following the removal of the needle, the temperature began to rise and the pulse became more rapid, though there had been no chill. January 18 , at 10 A.M. the temperature was 102 degrees; at 8 P.M. 103.5 degrees with a pulse of 140 . The face became somewhat dusky, the lips bluish, and the tongue took on a heavy brown coating. There were constipation and much restlessness. She was unable to sleep on the nights of January 18 and 19 . Up to January 21 , much the same general condition obtained. There remained the same cast of countenance. The pulse remained regular but rapid. Both heart sounds were audible. There was no dyspnea, but great restlessness. There was unusual mental alertness. The eyes were bright. There was sighing at times. She had no appetite and was almost free from pain. There was an increase of the precordial dulness. A diagnosis of septic pericarditis was made and an incision with flushing and drainage advised and declined. On January 21, the temperature subsided and the pulse went down to 109. This period of apparent improvement lasted until January 25 , when the temperature again began to rise and the area of precordial dulness to increase, indicating an increasing effusion. An operation was again urged, and, in view of the gravity of the case, accepted. Accordingly on Januaay 26 , with the able assistance of Dr. J. Vanderlaan and Dr. G. S. Williams, the wound for the extraction of the needle was enlarged, and an incision carried down through the intercostal muscle one and a half inches to the left of the sternum in the fifth interspace, keeping as nearly as could be to the point of entrance of the needle. A careful dissection soon carried us through the pericardium, which seemed to be attached to the anterior wall. About an ounce of thick pus and a considerable amount of puro-sanguinoform half clotted matter were evacuated. No effort was made to make extensive exploration, but the finger detected within the pericardium much soft fibrinous material which was easily broken down. The cavity was flushed with a saline solution and an iodoform wick inserted. No great shock was suffered. The temperature was normal on the evening of the day of the operation, with a pulse of 108, and never again went above 100 degrees. The patient made an uneventful recovery, being discharged cured just twenty-four days after the operation.

The extreme gravity of a case of purulent pericarditis warrants almost any measure that holds out hope of relief. When that measure is so well grounded a principle of surgery as that of the free evacuation of septic material whenever found, it is gratifying to find its application in practice followed by so encouraging results as a review of the preceding cases shows. Out of twelve cases here reported, some of which were 
complicated with diseases in themselves necessarily /Ewan's studies of mastoid disease and brain abscess, fatal, there were eight recoveries. It is my belief as Kraske's incision for removal of the rectum, are all that under proper precautions, pericardiotomy is results of anatomic studies applied to surgery; they atteaded by no greater danger than pleurotomy, and are what might be called anatomic surgery and they in cases unccomplicated by maladies tending toward demonstrate its value.

a fatal termination, the results will be equally as good. It is the only procedure which justifies itself to the modern surgeon. The dangers attending incision and drainage are not to be compared with the danger of expectant inactivity, and I am certain that many of them are overestimated. Pericardiotomy offers the only chance for safety in these cases, and must take its place among the operations sanctioned by good usage and good sense.

159 Jefferson Street.

\section{A NEW INCISION FOR THE SURGERY OF THE BILE TRACTS.}

BY A. D. BEVAN, M.D.

PROFESSOR OF ANATOMY, RUSH MEDICAL COLLEGE; SURGEON TO PRESBYTERIAN AND ST. LUKE'S HOSPITALS.
CHICAGO.

The subject of the surgery of bile tracts is of recent development. In 1867 Bobbs of Indiana performed cholecystotomy. In 1882 Langenbuch' removed the gall bladder, cholecystectomy. In the same year Gaston suggested cholecystenterostomy, which was later perfected by the introduction of the anastomosis button by Murphy. In 1890 Courvoisier performed and advocated choledochotomy, and later the removal of stones from the cystic duct and hepatic duct were reported.

In the last five years the value and possibilities of the surgery of the bile tracts has been so thoroughly demonstrated, that it is today a recognized field to all men doing abdominal work.

A few years ago, when I began to do surgical work on the bile tracts, I carefully reviewed the topographic anatomy of this region. I was surprised to find how completely the subject had been neglected by both anatomists and surgeons. And here let me say a word in behalf of surgical anatomy. It is the boast of some surgeons of the present brilliant aseptic period that they ignore anatomic boundaries and anatomic reasoning in their operative work. It is so easy to cut and ligate and suture deep and superficial structures and obtain primary union, that a careful study of operative procedures is regarded often as unnecessary, and is as a rule neglected.

In surgical training, for the time being, anatomy is overshadowed by pathology, and as a result, the surgeons of today are better pathologists to be sure, but much poorer anatomists than the surgeons of the past generation. This is a fault which is often evident even in the work of eminent surgeons, and which must be corrected. A perfect mastery of the surgical anatomy is absolutely necessary for the performance of the best operative surgery in any field of surgical labor. This applies with special force to the surgery of the bile tracts, and yet many operations on this region are performed by men who have no clear idea of the anatomic relations of the parts or of the possible dangers, and who do not see the necessity of preparing themselves by cadaver study before undertaking such work. Such brilliant work as Kocher's teachings of making incisions in the lines of normal cleavage, as Bassini's hernia operation, as McBurney's and McArthur's splitting operation for appendicitis, as Hartley's removal of the Gasserian ganglion, as Mc-ldanger to important structures, I undertook a series 